\title{
A Local Circuit Interaction in the Flight System of the Locust
}

\author{
R. Meldrum Robertson and David N. Reye ${ }^{a}$ \\ Department of Biology, McGill University, Montreal, Quebec H3A 1B1, Canada
}

\begin{abstract}
Most of the interneurons that have been identified in the locust flight system to date are spiking, intersegmental interneurons and the described interactions between them are spike-mediated postsynaptic potentials. We wished to discover whether the same interneurons also communicate via subthreshold interactions to form local circuits independent of their spike-mediated connections.

Using a deafferented flight preparation of the locust and glass microelectrodes, we recorded simultaneously from the neuropil segments of different interneurons within a single thoracic ganglion. Interneuron 301 had an indirect connection with the contralateral 301 and an indirect connection feeding back to itself. The feedback circuit could be activated with subthreshold stimuli. Spikes in a 301 affected self and contralateral partner, whereas subthreshold stimuli affected only self. Thus, this paper demonstrates the existence of 2 pathways from a single interneuron that can be functionally separate depending on whether activity in the interneuron is subthreshold or suprathreshold for spiking. The results show that dendritic regions of spiking intersegmental interneurons in the locust can participate in local circuits whose operation could have a considerable role to play in neural integration.
\end{abstract}

Insects have provided several excellent model systems for the study of the neural integrative processes underlying the generation of motor patterns and behavior. Indeed, much of the detailed knowledge of interactions mediated by graded release of transmitter from local, nonspiking interneurons has come from work with thoracic neurons in the locust (Burrows and Siegler, 1976, 1978; Burrows, 1985; Siegler, 1985). Interactions mediated by subthreshold or graded release of transmitter from spiking interneurons have been demonstrated in insects (Simmons, 1982), as well as in other systems (Raper, 1979; Graubard et al., 1983). Ultrastructural evidence indicating a mixing of input and output synapses on branches of spiking interneurons in the thoracic ganglia of locusts (Watson and Burrows, 1983, 1985) suggests a considerable role for local signal processing. However, such local integration has not been physiologically demonstrated. We have investigated the possibility that spiking

\footnotetext{
Received Nov. 10, 1987; revised Feb. 8, 1988; accepted Mar. 10, 1988.

We thank R. Chase, K. G. Pearson, and G. Pollack for their comments on a previous version of the manuscript. Supported by a grant from the Natural Sciences and Engineering Research Council of Canada and by the Faculty of Graduate Studies and Research of McGill University.

Correspondence should be addressed to R. M. Robertson at his present address: Department of Biology, Queens's University, Kingston K7L 3N6, Canada.

a Present address: Department of Neurobiology, Research School of Biological Sciences, Australian National University, Box 475, P.O., Canberra, A.C.T. 2601, Australia.

Copyright (C) 1988 Society for Neuroscience $0270-6474 / 88 / 103929-08 \$ 02.00 / 0$
}

interganglionic interneurons are capable of participating in local circuits which can function independently of the spiking activity of the interncurons via subthreshold release of transmitter in a single ganglion. We demonstrate here the operation of such a circuit among interneurons in the flight system of the locust.

In the locust there is a single pair of bilaterally symmetrical interneurons that have been given the identity number 301 and that have their somata in the mesothoracic ganglion (Robertson and Pearson, 1983). Each 301 has extensive branching in the mesothoracic ganglion and an axon that travels posteriorly in the connective contralateral to the soma to branch throughout the metathoracic ganglion before exiting in an abdominal connective. The postsynaptic potentials that have been described in some interneurons following each spike in 301 after a short ( $1 \mathrm{msec}$ or less after subtracting conduction delay) constant latency are all inhibitory. However, each spike in 301 is also followed in other interneurons by a longer-latency depolarizing potential. The response with the longer latency is reversed by hyperpolarizing the postsynaptic neuron and is therefore caused by a reduced conductance across the postsynaptic membrane. This property and others suggest that the synaptic mechanism for the delayed depolarization is a disynaptic disinhibition with tonic release of the inhibitory transmitter at the second synapse (Robertson and Pearson, 1985). We investigated the effect of spikes and the effect of subthreshold depolarizing pulses of current in one interneuron 301 , on the contralateral 301 , and on itself.

\section{Materials and Methods}

A deafferented preparation of the locust, Locusta migratoria, which was capable of expressing the centrally generated flight rhythm (Robertson and Pearson, 1982), was used for this study. Intracellular recordings were made from interneurons 301 using glass microelectrodes either filled at the tip with $4 \%$ Lucifer yellow and backfilled with $0.5 \mathrm{M}$ lithium chloride (electrode resistance $>100 \mathrm{M} \Omega$ ), or filled with $1 \mathrm{~m}$ potassium acetate (electrode resistance, 30-60 M $\Omega$ ). The neurons were penetrated in the neuropil segment which runs across the mesothoracic ganglion in the 5th dorsal commissure (Tyrer and Gregory, 1982). This was confirmed at the end of experiments in which the neurons were filled with Lucifer yellow, by visualizing the neurons before withdrawing the electrode using a Leitz epifluorescence illuminator attached to the dissection microscope. Conventional techniques were used to amplify and store the recorded signals and to pass current into the neurons.

The experiments described in this paper were performed in the course of general investigations of the circuit of flight interneurons undertaken during the past several years. The physiology and structure of interneuron 301 have been determined innumerable times $(n>100)$, and it was possible to identify 301 on the basis of its characteristic physiology alone. All the phenomena described here have been observed in at least 3 different preparations. Some preparations (approximately 20\%) failed to show the interactions to be described below. However, this is unsurprising considering the variability in the general level of excitability of different preparations (Robertson and Pearson, 1985) and the probability that the interactions are indirect. 

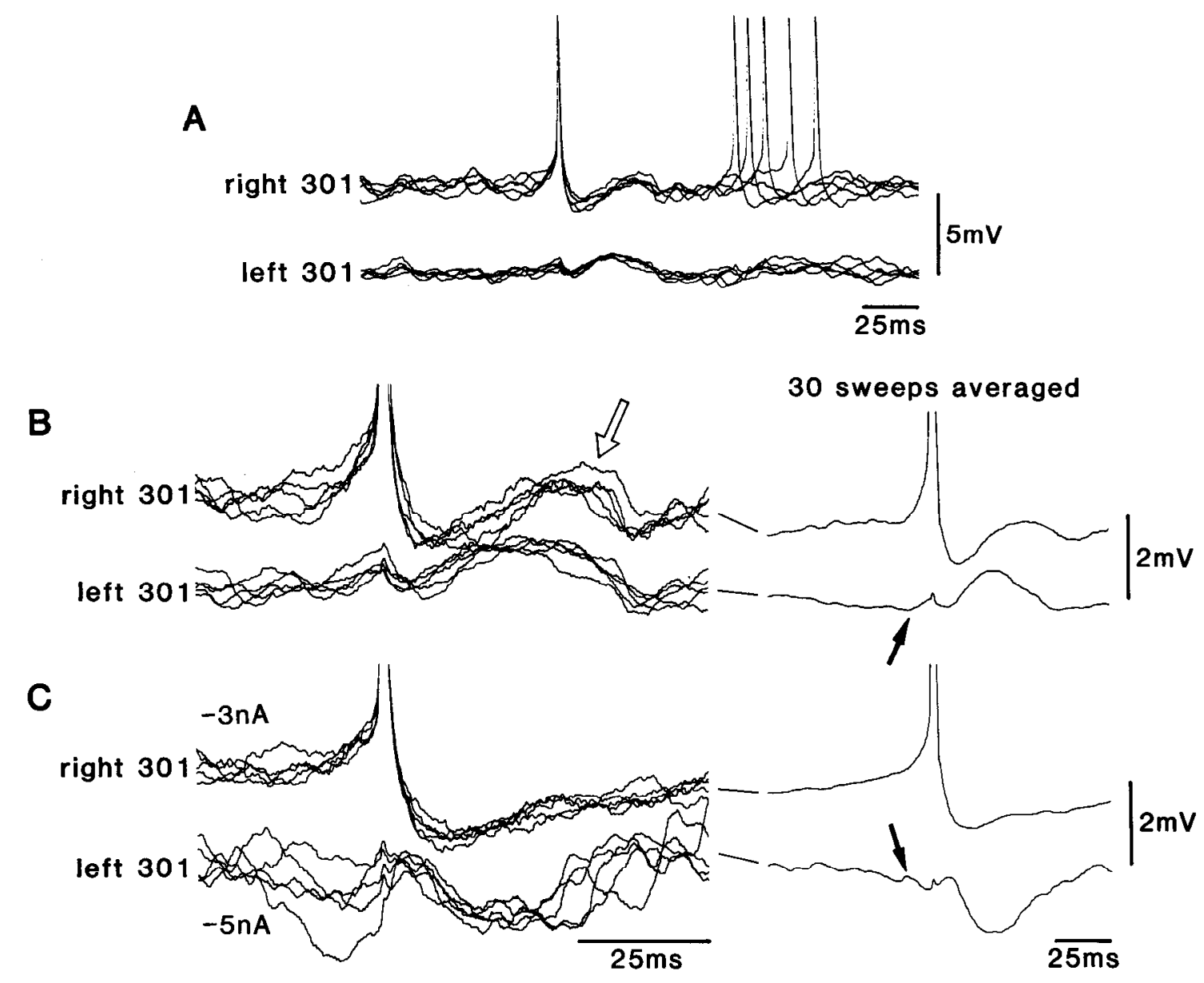

Figure 1. A, Simultaneous intracellular recordings from the right 301 and the left 301 . Five superimposed sweeps of the oscilloscope triggcred off the rising phase of the spike in the right 301 show that each spike in the right 301 was followed by a depolarizing potential in itself and a matching depolarizing potential in the left $301 . B$, As for $A$ but at greater amplification and faster sweep speed. Note the similarity between the potentials in right and left $301 \mathrm{~s}$ following each right 301 spike. The slight difference at the beginning of the potentials can be attributed to an effect of the spike after hyperpolarization in the right 301. Note also the flattened appearance at the peak of the potential and the rapid repolarization (open arrow). $C$. Hyperpolarization of right 301 with $-3 \mathrm{nA}$ of current abolished the postspike potential, while hyperpolarization of left 301 with -5 $\mathrm{nA}$ of current reversed the synaptic potential. The right 301 could not be hyperpolarized further without preventing it from firing spikes. The righthand portions of these figures show averaged traces of the potentials. These do not show the true shape of the potentials due to variability in the latency and duration of individual potentials. Note that the spike in right 301 tended to be initiated at the same time as a synaptic potential that is evident in the left 301 and that the latter was reversed by the hyperpolarizing current (solid arrows). The small depolarizing transient potential associated with the contralateral action potential is an artifact caused by capacitative coupling between the electrodes, and, therefore, it was not reversed by the hyperpolarizing current.

\section{Results}

The left 301 and the right 301 fired synchronous bursts of action potentials during expression of the flight rhythm. The synchrony was due to a large amount of common synaptic input. Direct chemical or electrical interconnections could not be detected. The neurons were, however, connected by reciprocal indirect connections. Each spike in one was followed by a delayed depolarization in the other (Fig. 1, $A, B$ ). Interestingly, single spikes in a 301 also induced a potential in itself similar in amplitude, shape, and duration to the potential induced in the contralateral partner (Fig. 1, $A, B$ ). The similarity between these potentials suggests that each spike in one 301 activated either an element of the flight circuitry presynaptic to both $301 \mathrm{~s}$, or similar but separate elements having equivalent effects on both $301 \mathrm{~s}$. These delayed depolarizations, in self and partner, had the properties of disynaptic disinhibitory connections such as have been previously described from 301 to other interneurons
(Robertson and Pearson, 1985). For example, they were reversed or abolished by the passage of hyperpolarizing current into the neuron from which the potential was recorded (Fig. 1C). In addition, they had a characteristic shape with a slow, almost exponential onset followed by a rapid repolarization and occasionally the peak of the potential had a flattened appearance (Fig. 1B). The latency from spike to onset of the potential varied between 4 and $6 \mathrm{msec}$, the duration between 25 and $45 \mathrm{msec}$, and the amplitude between 0.5 and $3 \mathrm{mV}$.

Stimulation of one of the interneurons with a pulse of depolarizing current subthreshold for the generation of spikes induced a depolarization in itself which outlasted the stimulus. By recording with 2 microelectrodes in the same neuron on either side of the ganglionic midline, it was possible to avoid the problems of artifact associated with recording and passing current through the same electrode. The electrode on the side ipsilateral to the cell body recorded action potentials of small amplitude and large synaptic potentials, indicating that it was 

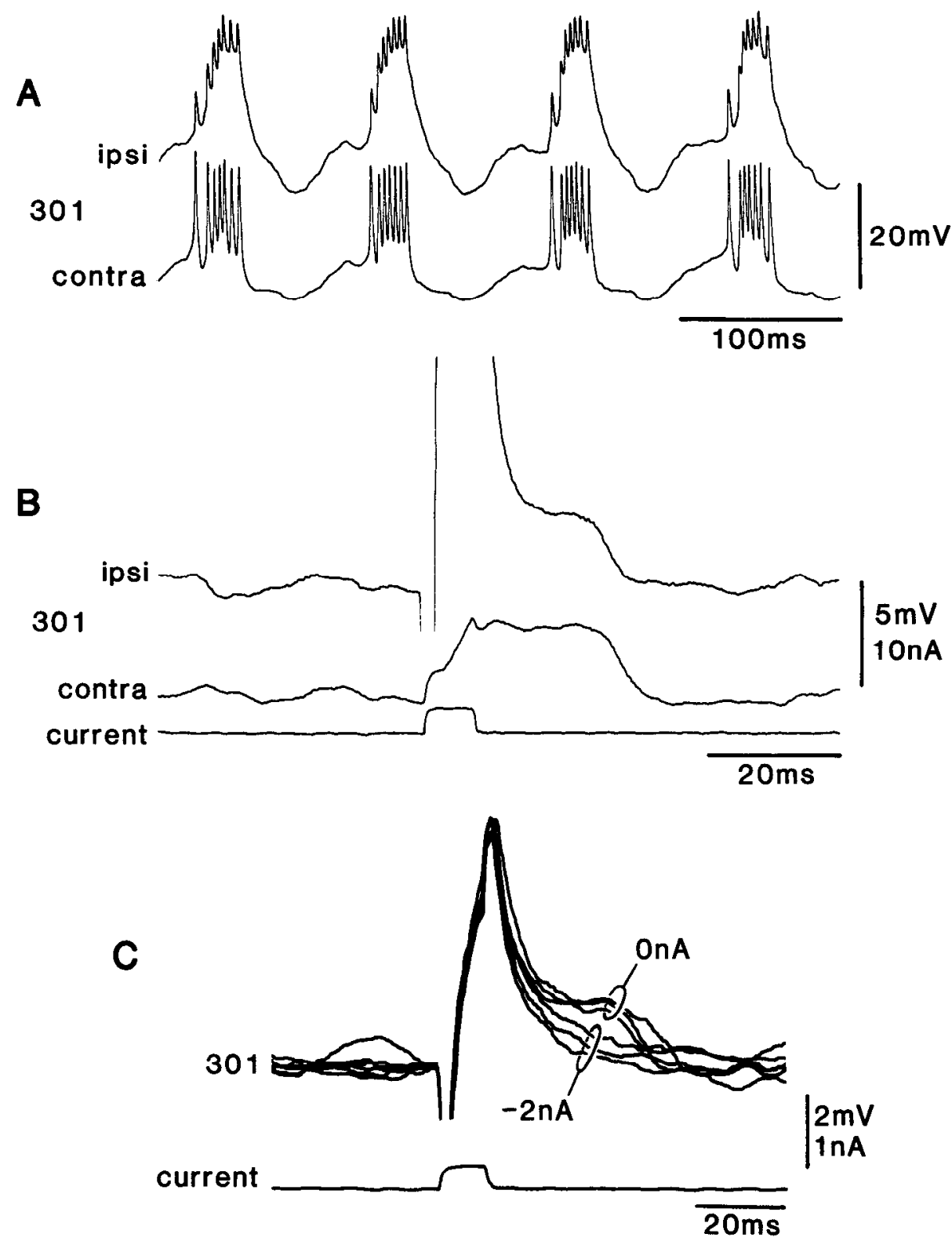

Figure 2. $A$, Simultaneous intracellular recordings from 2 positions in the neuropil segment of a single 301 interneuron during expression of the flight rhythm. The trace labeled $i p s i$ was from the electrode ipsilateral to the cell soma and the trace labeled contra from the electrode contralateral to the soma. Note that the ipsilateral electrode recorded action potentials of reduced amplitude and synaptic potentials of increased amplitude relative to the contralateral electrode, indicating that the ipsilateral electrode was in passive membrane close to the sites of synaptic input. $B$, A subthreshold depolarizing stimulus delivered through the ipsilateral electrode induced a depolarization that was approximately 4 times the duration of the stimulus. The trace from the ipsilateral electrode shows a large artifact due to an unbalanced bridge, while the trace from the contralateral electrode shows only small coupling artifacts associated with the beginning and end of the stimulus pulse. $C$, Intracellular recording and stimulation from a 301 with a single electrode. Six superimposed sweeps triggered by a subthreshold depolarizing stimulus pulse. For 3 of these sweeps no additional current was passed $(0 n A)$, and depolarizing potentials outlast the stimuli. For the remaining 3 sweeps, the membrane was hyperpolarized with continuous current $(-2 \mathrm{nA})$, and this abolished the depolarizing potential, allowing the membrane potential to repolarize exponentially. The position of each trace was adjusted so that it was at the same level at the start of each stimulus. The traces show artifacts associated with the beginning and end of each stimulus pulse. electrically distant from active membrane capable of supporting action potentials (Fig. $2 A$ ). Recordings from the electrode contralateral to the soma demonstrated that the stimulus applied across passive membrane through the ipsilateral electrode was subthreshold for action potential generation and that it induced a long-lasting depolarization approximately 4 times the duration of the stimulus (Fig. $2 B$ ). The peak of the potential was flattened, giving it a plateau-like appearance. The latency to onset could not be determined due to the inability to separate the phenomenon from the direct (passive) response of the membrane to the stimulus. The duration varied between 20 and $50 \mathrm{msec}$. The amplitude of the potential was variable (see below) but never exceeded $4 \mathrm{mV}$. The simplest interpretation of this phenomenon is that the subthreshold stimulus was capable of activating the same synaptic pathway to itself that was activated by spikes. Consistent with this interpretation is the observation that the long-lasting potential is similarly abolished by the passage of hyperpolarizing current ( $\mathrm{Fig}$. 2C). Also, we did not observe any difference in the characteristics (e.g., amplitude, duration, ra- pidity of repolarization) of the spike-mediated potential and the long-lasting potential, which were great enough to suggest that the 2 potentials were produced by different mechanisms. Differences between the initial portions of the 2 potentials would be unavoidable due to the contribution of the spike after hyperpolarization in one and the contribution of the direct effect of the current stimulus in the other. The rapid repolarization at the end of the long-lasting depolarization could be attributed to the occurrence of spontaneous IPSPs following spikes of different flight interneurons (e.g., interneuron 501; Fig. 3). The amplitude of spontaneous IPSPs appeared enhanced if they occurred within approximately $50 \mathrm{msec}$ following the subthreshold stimulus (Fig. 3, $B, C$ ). There was a strong correlation of the occurrence of IPSPs with a return to the baseline membrane potential such that we can rule out unrelated coincidence of the 2 events. However, we cannot distinguish between the possibilities, first, that the occurrence of an IPSP triggered a repolarization greater than its own amplitude and, second, that the IPSP amplitude was really greater and its duration merely out- 
A
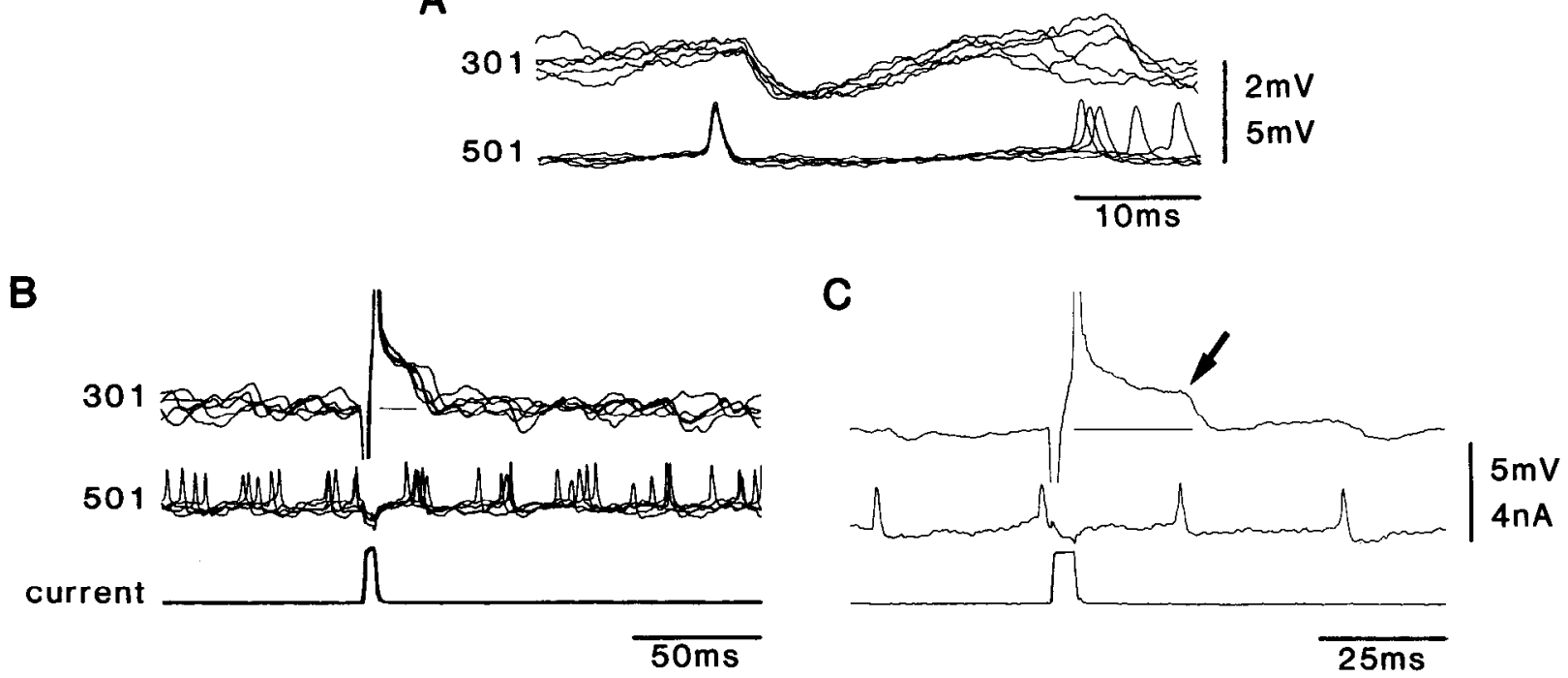

Figure 3. A, IPSPs recorded in a 301 a short and constant latency after each spike recorded in 501. Five superimposed sweeps triggered off the rising phase of the presynaptic spike in 501. B. Simultaneous intracellular recordings from 301 and 501. Five superimposed sweeps triggered off a subthreshold stimulus delivered to 301. The position of each 301 trace was adjusted so that it was at the same level at the start of each stimulus. Note that a long-lasting depolarization in 301 following each stimulus is terminated by the occurrence of an IPSP, which thus contributes to the rapidity of the repolarization phase. $C$, A single set of traces from $B$ shown at a faster sweep speed demonstrates that the amplitude of an IPSP occurring $25 \mathrm{msec}$ from the start of the stimulus was greatly increased (solid arrow). The horizontal lines drawn on the 301 traces of $B$ and $C$ indicate the membrane potential levels prior to the stimulus. The traces show artifacts associated with the beginning and end of each stimulus pulse.

lasted the duration of the depolarization, thus masking the termination of the latter.

The long-lasting depolarization was variable in its expression both within a single preparation and between animals. It was often difficult to detect among the large amount of spontaneous synaptic activity which was evident in neuropil recordings taken from 301 . In some cases, the phenomenon could be revealed by the observation of a 40-50 msec period following the subthreshold stimulus when spikes in 301 were more likely to occur (Fig. 4). With stimuli of constant amplitude, the amplitude of the response was variable and did not depend in any obvious way on the membrane potential of the neuron (Fig. $5 A$ ). It was not possible to demonstrate a graded effect in the response to subthreshold stimuli of different strengths. Gradually increasing the strength of long-duration current pulses demonstrated a threshold for an abrupt change in the trajectory of the membrane potential response (Fig. $5 B$ ). The increase of the amplitude of the membrane potential response was small, and past this threshold the membrane responded in a linear fashion to continued increases in the amplitude of the stimuli (not shown). Numcrous cxpcriments $(n>10)$ investigating the voltage responses of a 301 to current pulses, using a single electrode and the discontinuous current-clamp circuitry of an Axoclamp electrometer (Axon Instruments Inc.) failed to reveal any other evidence indicating nonlinearity of the membrane.

Although action potentials in one interneuron 301 produced similar synaptic potentials in both $301 \mathrm{~s}$, subthreshold stimuli were effective in producing long-lasting depolarizations of only the 301 being stimulated. Stimulation of one interneuron 301 with a pulse of depolarizing current sufficient to cause it to fire a burst of spikes induced a long-lasting depolarization in the contralateral 301 and in itself(Fig. 6A). Stimulation of the same interneuron with a pulse of depolarizing current subthreshold for spiking was effective in producing the long-lasting depolar- izing potential in itself but could no longer affect the contralateral 301 (Fig. $6 B$ ). In separate experiments, subthreshold stimulation of a 301 was never seen to affect the contralateral 301 , whereas in all cases a train of action potentials in a 301 caused a depolarization of the contralateral 301 .

\section{Discussion}

The results presented here clearly show that each 301 had an indirect synaptic connection with its contralateral partner. The PSP was reversed by hyperpolarizing the postsynaptic neuron, indicating that it was caused by a decreased conductance across the postsynaptic membrane. This characteristic and others (latency, time course, shape) suggest that the PSP was caused by a disynaptic disinhibitory connection between the $2301 \mathrm{~s}$, similar to connections that have been described from a 301 to other interneurons (Robertson and Pearson, 1985). The slowness of the onset can thus be attributed to the PSPs being caused by a transient reduction or termination of a hyperpolarizing conductance, which would result in a passive recovery to a new steady membrane potential, whereas the flattened peak of the PSPs can be attributcd to the membranc potential of the intercalated neuron (causing the hyperpolarizing conductance) passing beneath a threshold for transmitter release. The results cannot distinguish between the possibilities that the hyperpolarizing conductance was caused by tonic graded release or tonic spiking activity of a presynaptic inhibitory interneuron. However, the rapidity of the repolarization does suggest the occurrence of a spike-mediated inhibitory postsynaptic potential whether or not graded release is also making a contribution to the resumption of the hyperpolarizing conductance.

Action potentials in a 301 produced depolarizing potentials in itself which were very similar to the PSPs induced in the contralateral 301. The similarity justifies the interpretation either that each spike in a 301 inhibited an interneuron which was 


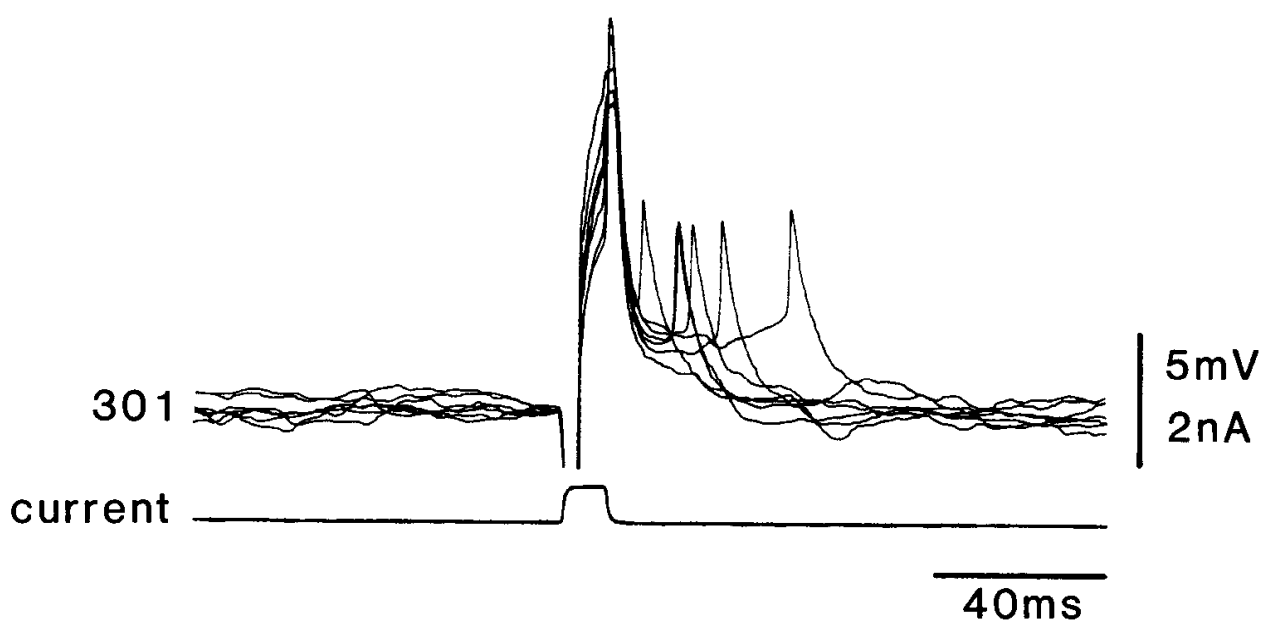

Figure 4. Subthreshold stimulation of a 301 evokes a period greatly outlasting the stimulus during which spikes are more likely to occur. Seven superimposed sweeps triggered off the stimulus. The position of each trace was adjusted so that it was at the same level at the start of each stimulus. Note the occurrence of spikes in 301 during the period 40-50 msec following each stimulus. No spikes can be discerned during the stimulus. The traces show artifacts associated with the beginning and end of each stimulus. presynaptic to both $301 \mathrm{~s}$ or that each spike in a 301 inhibited 2 equivalent interneurons (e.g., a bilaterally symmetrical pair of interneurons) each of which was presynaptic to one 301 . Thus, it is reasonable to conclude that there exists a synaptic circuit from a 301 feeding back onto itself.

The most intriguing result presented here is that a subthreshold depolarizing stimulus delivered to a 301 induced a depolarization in itself which far outlasted the duration of the stimulus. The simplest interpretation of this phenomenon is that subthreshold changes in membrane potential caused release of transmitter from 301 and activated the same feedback circuit to itself that was activated by spikes. An alternative interpre- tation is that the long-lasting potential was a result of a voltagedependent endogenous property of the membrane that could be terminated by the occurrence of an IPSP. Although it is not possible to rule out this interpretation directly, it is highly unlikely. In addition to its similarities with the synaptic phenomenon (see above), the potential induced by a subthreshold stimulus showed none of the properties commonly attributed to slow potentials generated by endogenous properties of the membrane, with the exception that it outlasted the duration of the stimulus. In other systems, actively generated plateau-like potentials tend to have a large amplitude and a long duration and to be all-ornone regenerative phenomena, and their expression is usually

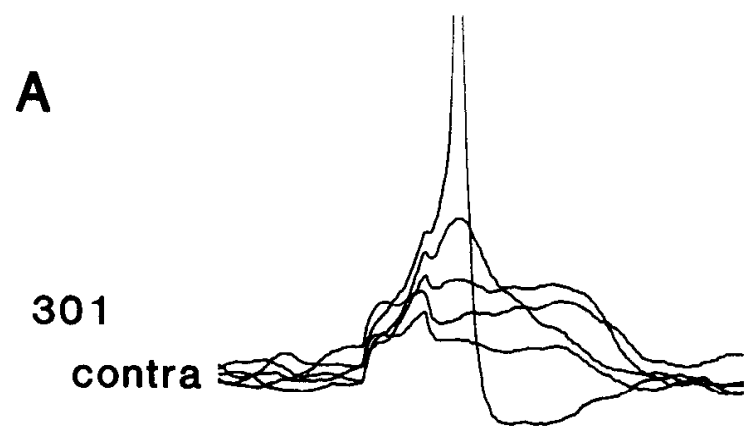

current

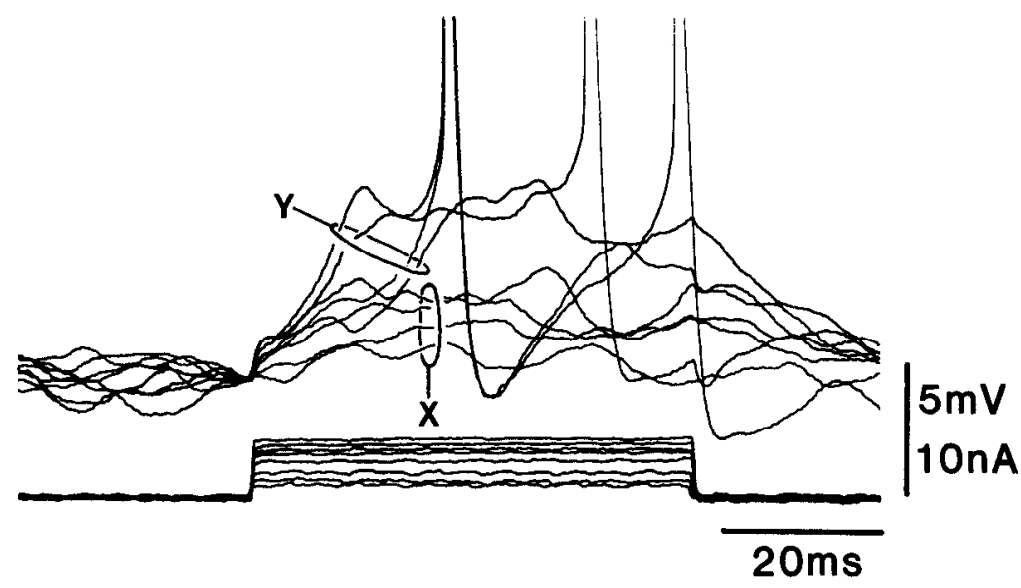

Figure 5. $A$, Intracellular recording and stimulation of a 301 with separate electrodes. Five superimposed sweeps triggered off a depolarizing stimulus pulse. The amplitude of the stimulus was the same for each trial, but the response varied considerably from a slight depolarization, through larger depolarizations longer than the stimulus and an aborted spike, to a full action potential. Note that there is no indication of an all-or-none, regenerative response and that the variability did not depend in any obvious way on the membrane potential of the neuron. In this series of traces the positions of the traces were not adjusted, and thus changes of level represent true changes of the membrane potential. $B$, Eight superimposed sweeps triggered from long depolarizing stimuli. The amplitude of the current stimuli was increased gradually but the response of the membrane showed an abrupt transition when the trajectory of the membrane potential became steeper (compare traces labeled $X$, for the 4 lowest amplitude stimuli, and traces labeled $Y$, for the 4 highest amplitude stimuli). The positions of the traces were adjusted so that each was at the same level at the beginning of the stimulus. The traces from the ipsilateral electrode have been omitted for clarity, although the stimuli were all delivered through this electrode. 


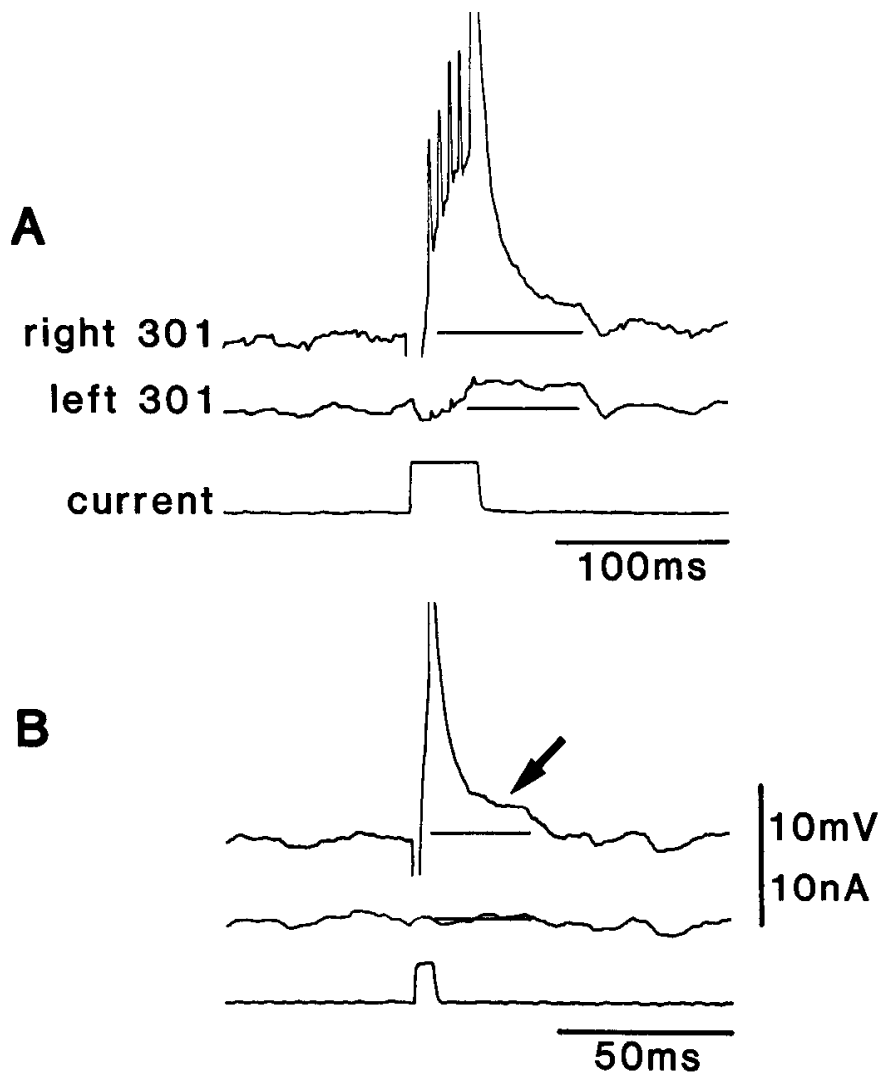

Figure 6. A, Simultaneous intracellular recordings from the right and left $301 \mathrm{~s}$. A suprathreshold depolarizing current pulse delivered to one 301 induced a long-lasting depolarization in itself and in the other 301. $B$, A subthreshold depolarizing current pulse delivered to one 301 induced a long-lasting depolarization in itself (arrow) but not in the other 301. The traces for the right 301 show large artifacts associated with the beginning and end of the current pulse and the bridge was not accurately balanced. The horizontal lines indicate the membrane potential levels prior to the stimulus. Note the different time scales in $A$ and $B$.

voltage dependent (Russell and Hartline, 1978; Tazaki and Cooke, 1979; Benson and Cooke, 1984). Slow regenerative potentials distinct from the sodium action potential have been recorded from insect neurons, but they have been shown only in active membrane after pharmacological manipulation (Yawo et al., 1985; Hochner and Spira, 1986; Blagburn and Sattelle, 1987). The long-lasting potential described here was small. It was induced by stimulation of passive membrane. It was variable in its expression, with no indication that it was an all-ornone, regenerative phenomenon. Its occurrence was not dependent on the membrane potential of the neuron in any obvious way. No other nonlinearity of the membrane could be revealed. Thus, the evidence overwhelmingly favors the interpretation that the long-lasting potential results from synaptic feedback via subthreshold release of transmitter from interneuron 301.

Any model of the circuitry between the $2301 \mathrm{~s}$ must also account for the observation that whereas spikes in a 301 affected self and partner, subthreshold stimuli affected only self. Figure 7 presents 2 model circuits, each of which can account for all the phenomena reported here. We have no evidence favoring one of the model circuits over the other. Only 2 further assumptions need be made. First, it must be assumed that subthreshold changes in membrane potential decay significantly
A.

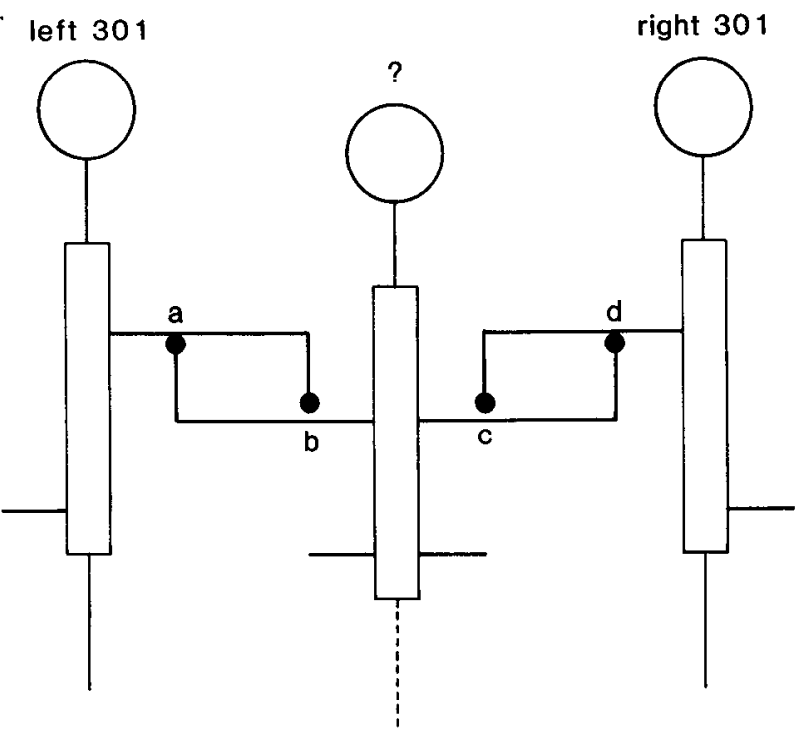

B.

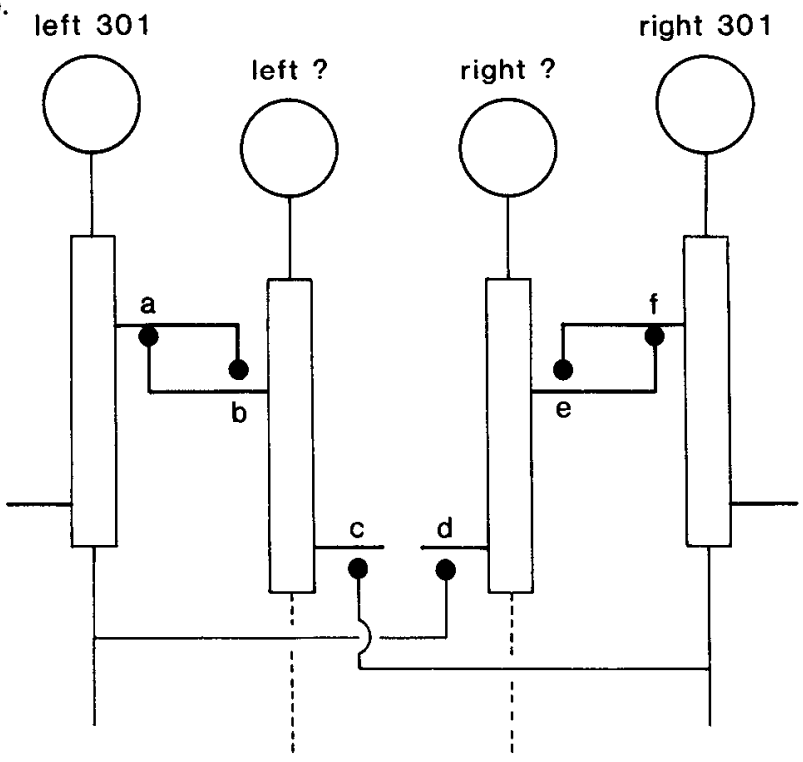

Figure 7. Two model circuits $(A$ and $B$ ) that can account for the phenomena described in this paper. Circles represent the cell somata of the interneurons. Long rectangles represent the neuropil segments of each neuron, with the lateral branches as dendritic branches and the descending lines as the axons. For the unknown neurons ("?"), the descending lines are dashed to indicate that these neurons may or may not be intersegmental interneurons. Filled circles represent inhibitory synapses. The elements of the synapses at $a$ and $d$ in $A$ and at $a$ and $f$ in $B$ are shown as contiguous to indicate the necessity for tonic release at these synapses (see text for further description).

with increasing distance of electrotonic spread. This is not controversial, and Siegler (1984) describes modeling studies demonstrating that the attenuation of signals throughout the neuropil branches of an insect interneuron can be substantial. Second, it must be assumed that although the intercalated interneuron is tonically releasing transmitter at its baseline potential, there is a threshold for transmitter release beneath which the connection is ineffective and larger inputs do not change the magnitude of 
the synaptic effect. This is similarly not controversial. Upper and lower thresholds establishing variable ranges of graded transmitter release have been described in locust thoracic interneurons (e.g., Burrows, 1985), and if the tonic hyperpolarizing conductance in a 301 resulted from tonic spiking in the intercalated interneuron, this lower threshold would simply represent a lack of spiking in the interneuron. Moreover, the flattened appearance of the peaks of the potentials lends some support to the idea.

The operation of the circuit in Figure $7 A$ can be described as follows. Synapses a and d from "?" to left and right $301 \mathrm{~s}$ are tonically releasing inhibitory transmitter. Thus, an action potential in the left 301 disynaptically disinhibits itself (through synapses $b$ and a) and the contralateral 301 (through synapses $b$ and $d$ ). The magnitudes of the PSPs produced in the $2301 \mathrm{~s}$ are similar because, despite attenuation of the IPSP throughout "?", it is large enough to hyperpolarize the membrane potential below threshold for release at both sites ( $a$ and $d$ ). The hyperpolarization below threshold can be markedly different at the 2 sites without affecting the sizes of the PSPs in both 301 s (i.e., far below threshold at a and just below threshold at d). A subthreshold depolarization of the left 301 affects itself and not the contralateral 301 because the smaller IPSP produced in "?" via subthreshold release at synapse $b$ is sufficient to take the membrane potential of "?" just below threshold for release at a but is too attenuated to have an appreciable effect on the membrane potential of "?" at d.

The operation of the circuit in Figure $7 B$ can be described as follows. Synapse a from left "?" to left 301 and synapse f from right "?" to right $\mathbf{3 0 1}$ are tonically releasing inhibitory transmitter. Thus, an action potential in the left 301 disynaptically disinhibits itself (through synapses $b$ and a) and the contralateral 301 (through synapses $d$ and $f$ ). The magnitudes of the PSPs produced in both $301 \mathrm{~s}$ are similar because a similar IPSP is produced in equivalent interneurons (left and right "?"). A subthreshold depolarization of the left 301 delivered relatively close to $b$ affects itself and not the contralateral 301 because either the subthreshold stimulus is too attenuated to have an appreciable effect at synapse $d$ or the thresholds for release at synapse $b$ and synapse $d$ are different.

Both of the model circuits predict that a spontaneous IPSP occurring during the long-lasting potential (i.c., within 40-50 msec after delivery of a subthreshold depolarizing stimulus) would have an increased amplitude. Thus, the results shown in Figure 3 can be accounted for because, first, the membrane potential of 301 was further from the reversal potential of the IPSP, and, second, the long-lasting potential was caused by a decreased conductance (i.e., the IPSP occurred during a period of increased membrane resistance).

It is unfortunate that we were unable to demonstrate a graded relationship between the strength of the stimulus and the amplitude of the resulting PSP. Indeed, even with a constant stimulus, the size of the response varied considerably. However, it is important to consider that, according to our models, the connection is disynaptic and, thus, that changes of the membrane potential of the intercalated neuron(s) (which could not be monitored) would affect the size of the response in a 301. It is likely that, as with other flight interneurons, the intercalated neuron(s) received a continuous and complex barrage of synaptic input. Transient variations in this input would affect the amplitude of evoked PSPs in 301 in an unpredictable manner in our experiments.
The functional significance of the connections described here is difficult to imagine given the current state of our knowledge of the operation of the flight circuit. The change in the trajectory of the membrane potential response of 301 (Fig. $5 B$ ) may play a part in increasing the speed of depolarization of 301 in response to an excitatory input. However, it is important to remember that the connections are described here in an inactive flight circuit. Activation of the flight circuit causes large membrane potential oscillations in the $301 \mathrm{~s}$, and this is likely to be true for the membrane potential(s) of the unknown neuron(s). Also, the general level of polarization around which the membrane potentials oscillate is set at different levels in different flight interneurons (Robertson and Pearson, 1984). Conceivably the connections described here have a more significant effect during expression of the flight rhythm. Determining the precise role of these interactions in an active circuit will take some time.

More important, however, and accepting the fact that the long-lasting depolarization is a synaptic phenomenon, the results demonstrate that a spiking interganglionic interneuron in an insect CNS can release transmitter as a consequence of a subthreshold stimulus delivered across passive membrane in the region of the interneuron where most synaptic integration occurs. The output synapses activated by such a stimulus are functionally isolated from the output synapses on the axonal branching in electrically distant ganglia. Even in the same ganglion, one pathway from a 301 (to the contralateral 301) is dependent upon the generation of spikes for its activation, whereas another (the feedback circuit to itself) is not. This shows the functional separation of pathways from the same neuron depending on the nature of that neuron's electrical activity. The separation within a single ganglion could be effected simply by the degradation of electrotonic signals with distance (Nelson et al., 1975). Alternatively, the output synapses of a single neuron could have different release properties (low and high threshold) and be segregated according to the identity of the postsynaptic neuron, with one population being concerned with local integration and another with driving followers. Resolution of this question must wait until the other interneurons in the pathways have been identified.

\section{References}

Benson, J. A., and I. M. Cooke (1984) Driver potentials and the organization of rhythmic bursting in crustacean ganglia. Trends Neurosci. 7: 85-91.

Blagburn, J. M., and D. B. Sattelle (1987) Calcium conductance in an identified cholinergic synaptic terminal in the central nervous system of the cockroach. J. Exp. Biol. 129: 347-364.

Burrows, M. (1985) Nonspiking and spiking local interneurons in the locust. In Model Neural Networks and Behavior, A. I. Selverston, ed., pp. 109-125, Plenum, New York.

Burrows, M., and M. V.S. Siegler (1976) Transmission without spikes between locust interneurones and motoneurones. Nature 262: 222224.

Burrows, M., and M. V.S. Siegler (1978) Graded synaptic transmission between local interneurones and motor neurones in the metathoracic ganglion of the locust. J. Physiol. (Lond.) 285: 231-255.

Graubard, K., J. A. Raper, and D. K. Hartline (1983) Graded synaptic transmission between identified spiking neurons. J. Neurophysiol. 50 : 508-521

Hochner, B., and M. E. Spira (1986) Two distinct propagating regenerative potentials in a single ethanol-treated axon. Brain Res. 398: 164-168.

Nelson, R., A. v. Lutzow, H. Kolb, and P. Gouras (1975) Horizontal cells in cat retina with independent dendritic systems. Science 189 : 137-139. 
Raper, J. A. (1979) Nonimpulse-mediated synaptic transmission during the generation of a cyclic motor program. Science 205: 304-306.

Robertson, R. M., and K. G. Pearson (1982) A preparation for the intracellular analysis of neuronal activity during flight in the locust. J. Comp. Physiol. 146: 311-320.

Robertson, R. M., and K. G. Pearson (1983) Interneurons in the flight system of the locust: Distribution, connections and resetting properties. J. Comp. Neurol. 215: 33-50.

Robertson, R. M., and K. G. Pearson (1984) Interneuronal organization in the flight system of the locust. J. Insect Physiol. 30: 95-101.

Robertson, R. M., and K. G. Pearson (1985) Neural circuits in the flight system of the locust. J. Neurophysiol. 53: 110-128.

Russell, D. F., and D. K. Hartline (1978) Bursting neural networks: A reexamination. Science 200: 453-456.

Siegler, M. V. S. (1984) Local interneurones and local interactions in arthopods. J. Exp. Biol. 112: 253-281.

Siegler, M. V. S. (1985) Nonspiking interneurons and motor control in insects. Adv. Insect Physiol. 18: 249-304.
Simmons, P. J. (1982) Transmission mediated with and without spikes at connexions between large second-order neurones of locust ocelli. J. Comp. Physiol. 147: 401-414.

Tazaki, K., and I. M. Cooke (1979) Isolation and characterization of slow depolarising responses of cardiac ganglion neurons in the crab, Portunus sanguinolentus. J. Neurophysiol. 42: 1000-1021.

Tyrer, N. M., and G. E. Gregory (1982) A guide to the neuroanatomy of locust suboesophageal and thoracic ganglia. Phil. Trans. R. Soc. London [Biol.] 297: 91-123.

Watson, A. H. D., and M. Burrows (1983) The morphology, ultrastructure and distribution of synapses on an intersegmental interneurone of the locust. J. Comp. Neurol. 214: 154-169.

Watson, A. H. D., and M. Burrows (1985) The distribution of synapses on the two fields of neurites of spiking local interneurones in the locust. J. Comp. Neurol, 240: 219-232.

Yawo, H., H. Kojima, and M. Kuno (1985) Low-threshold, slowinactivating $\mathrm{Na}^{+}$potentials in the cockroach giant axon. J. Neurophysiol. 54: 1087-1100. 